

\title{
Influence of the laser light absorption by the colloid on the properties of silver nanoparticles produced by laser ablation in stirred and stationary liquid
}

\author{
A. Resano-Garcia, Y. Battie, A. Koch, A. En Naciri, N. Chaoui
}

\section{- To cite this version:}

A. Resano-Garcia, Y. Battie, A. Koch, A. En Naciri, N. Chaoui. Influence of the laser light absorption by the colloid on the properties of silver nanoparticles produced by laser ablation in stirred and stationary liquid. Journal of Applied Physics, 2015, 117 (11), pp.113103. 10.1063/1.4915277 . hal01517450

\section{HAL Id: hal-01517450 \\ https://hal.univ-lorraine.fr/hal-01517450}

Submitted on 11 May 2017

HAL is a multi-disciplinary open access archive for the deposit and dissemination of scientific research documents, whether they are published or not. The documents may come from teaching and research institutions in France or abroad, or from public or private research centers.
L'archive ouverte pluridisciplinaire HAL, est destinée au dépôt et à la diffusion de documents scientifiques de niveau recherche, publiés ou non, émanant des établissements d'enseignement et de recherche français ou étrangers, des laboratoires publics ou privés. 
Influence of the laser light absorption by the colloid on the properties of silver nanoparticles produced by laser ablation in stirred and stationary liquid

A. Resano-Garcia, Y. Battie, A. Koch, A. En Naciri, and N. Chaoui

Citation: Journal of Applied Physics 117, 113103 (2015); doi: 10.1063/1.4915277

View online: http://dx.doi.org/10.1063/1.4915277

View Table of Contents: http://aip.scitation.org/toc/jap/117/11

Published by the American Institute of Physics

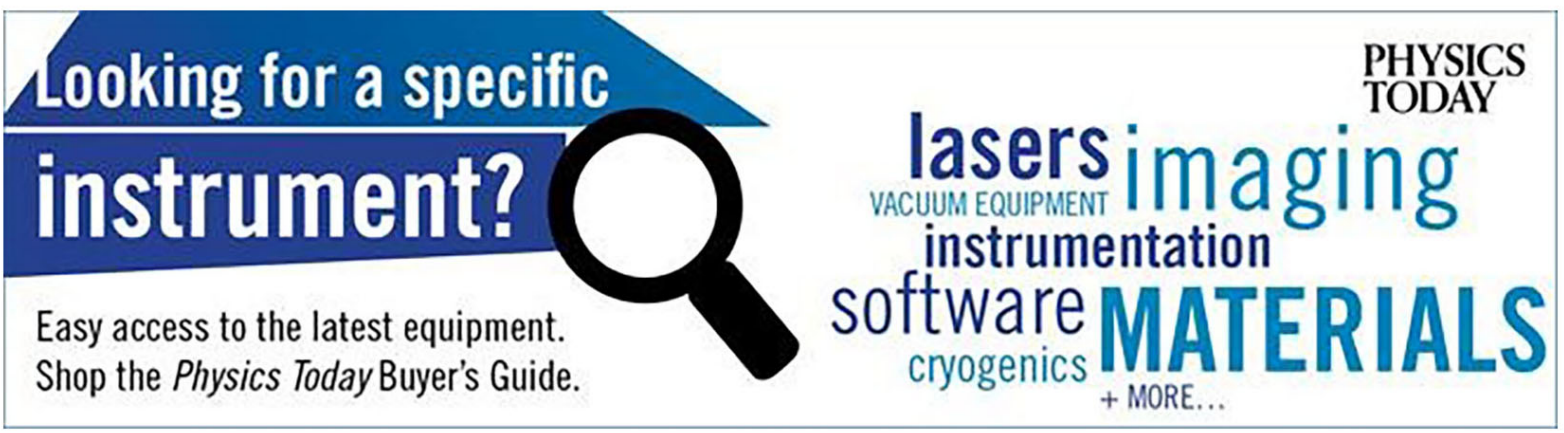




\title{
Influence of the laser light absorption by the colloid on the properties of silver nanoparticles produced by laser ablation in stirred and stationary liquid
}

\author{
A. Resano-Garcia, Y. Battie, A. Koch, A. En Naciri, and N. Chaouia) \\ LCP-A2MC, Institut Jean Barriol, Université de Lorraine, 1, Bd Arago, 57070 Metz, France
}

(Received 12 December 2014; accepted 6 March 2015; published online 18 March 2015)

\begin{abstract}
Silver nanoparticles were produced by nanosecond pulsed-laser ablation at $1064 \mathrm{~nm}$ of $\mathrm{Ag}$ in pure water. These experiments were performed using an alternative ablation cell design where a cylindrical shaped Ag target was horizontally irradiated, while the liquid was stirred by a stir rod coaxially arranged to the target. The repeatability of the generated colloids properties (extinction and size distribution) is assessed by statistical tools. The colloids properties prepared under stationary liquid are found to be unpredictable, while they are highly repeatable at high stirring speed. At the same time, electronic microscopy examinations of the irradiated Ag targets revealed that the width of the laser-machined grooves exponentially decays in stationary liquid and almost linearly under high stirring speed as the ablation proceeds. In the latter case, the decay rate was found to be constant from one experiment to the other, while it was not repeatable stationary liquid. We show that the decay of the groove width is due to an attenuation of the laser energy reaching the target surface due to the formation of a more or less dense NPs layer in front of the target as the ablation proceeds. Using the ablation time-dependence of the groove width, we can quantify the attenuation factor of the laser energy with exposure time. Finally, the relationship between the laser energy attenuation, stirring speed, and repeatability of the colloids properties is interpreted and discussed in terms of mass transfer. (C) 2015 AIP Publishing LLC. [http://dx.doi.org/10.1063/1.4915277]
\end{abstract}

\section{INTRODUCTION}

Nanoparticles (NPs) of noble metals (Ag and $\mathrm{Au}$ ) have attracted great interest from researchers because of their potential applications in various fields such as biomedicine, ${ }^{1,2}$ catalysis, ${ }^{3}$ surface-enhanced spectroscopy, ${ }^{4}$ or biochemical sensing. ${ }^{5}$ Conventional preparation routes of metal NPs are often based on reduction of a metal salts in presence of stabilizing ligands. However, the main drawback of these procedures is the presence of contaminants on the surface of the NPs which is problematic when high surface activity is required for applications such as catalysis and biomedicine. Pulsed laser ablation in liquid ${ }^{6,7}$ (PLAL) is an alternative fabrication method that addresses this drawback and helps to produce pure colloids, i.e., free of chemical agents as long as a pure metal target is ablated in pure solvent. Several other advantages of PLAL can be mentioned such as its possibility of producing naturally stable colloids because of partial surface oxidation ${ }^{8}$ and its versatility since it is not dependent on the availability of a chemical precursor but allows, in principle, to produce NPs of any material in any transparent liquid.

However, the control of size distribution of the NPs is of the major issues of PLAL in pure solvents. ${ }^{7}$ These characteristics are related to processing parameters including both laser parameters (wavelength, pulse duration, fluence, laser spot area, repetition rate, experiment duration, etc.) and ablation cell parameters (irradiation geometry, target geometry and surface quality, solvent volume, distance covered by the

\footnotetext{
a) Author to whom correspondence should be addressed. Electronic mail: nouari.chaoui@univ-lorraine.fr.
}

laser beam in the solvent, stirring, etc.). Furthermore, the different experimental conditions from the literature and the interdependence of several processing parameters also raise problems of reproducibility rendering often difficult the process optimisation on the basis of literature results. ${ }^{5}$ The efforts towards optimization and control of the process have been mostly dedicated to effects of laser parameters, like wavelength, pulse duration, energy and focusing conditions on productivity, and size distribution of the colloids. ${ }^{9-11}$ Much less effort has been focused on ablation cell design. On one hand, the simplest and most frequent design encountered in the literature, consists in irradiating in a vertical direction a metal target placed at the bottom of a vessel filled with liquid through the air-liquid interface. ${ }^{7-11}$ Usually, the liquid layer over the target is fixed to several millimetres to limit the absorption of the laser by the NPs. The laser beam is focused on the target using appropriate optics to produce surface ablation and generation of NPs. Generally, the vessel is mounted on a moving stage in order to refresh the target surface during ablation procedure and avoid excessive digging and/or heating of the target, especially at high repetition rate. The vessel motion may produce perturbations of the wave front of the laser due to oscillation of liquid surface (vibration, ripples, tilt, etc.) causing repeatability problems. ${ }^{12}$ Additionally, the distance covered by the laser in the liquid, which has a significant influence on NPs size distribution, might change during the ablation procedure especially when volatiles solvents are used. ${ }^{13}$

On the other hand, several authors ${ }^{12-17}$ have adopted a horizontal configuration in which the metal target is horizontally irradiated through the side of a transparent vessel filled 
(a)

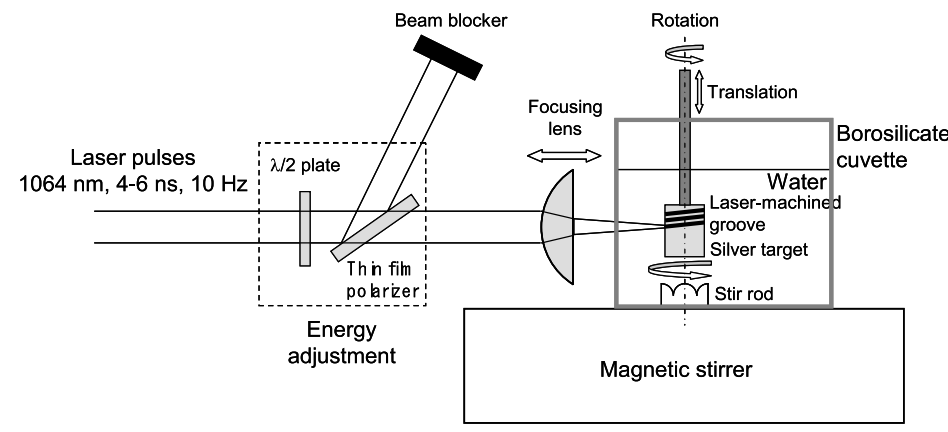

(b)

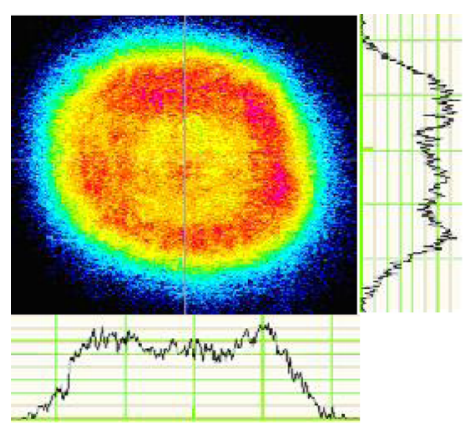

FIG. 1. (a) Schematic diagram of the experimental setup used to generate Ag colloids by Pulsed-laser Ablation in Liquid (b) Near-field spatial profiles of the laser beam. The diameter of the laser beam is $5 \mathrm{~mm}$.

with liquid. For instance, Prochàzka et al. ${ }^{14}$ used an Ag foil vertically immersed in the centre of a quartz cell filled with $7 \mathrm{ml}$ and horizontally irradiated by an Nd-YAG laser (20 ns and $1064 \mathrm{~nm}$ ). Sobhan et al. ${ }^{12}$ used a similar configuration in fs-regime ablation experiments, but the cell is fixed on a three-axis moving stage. This configuration overcomes the problems of liquid surface motion and ensures a precise control and constancy of the distance covered by the laser in the liquid, which play a significant role in both productivity and size distribution of NPs. ${ }^{12,13}$

In addition, the ablation experiment is usually conducted in stirred liquid using a simple magnetic stirrer or a more sophisticated liquid flow system. ${ }^{18}$ Prochàzka et al. ${ }^{14}$ showed that liquid stirring upon the ablation procedure enables an increase of the ablation yield and a reduction of the mean particles size. They explained these results by an increase of the amount of NPs crossing the laser beam which promotes laser-induced fragmentation. Barcikowski et al., ${ }^{18}$ using ultrashort pulse (ps and fs) at high repetition rate, showed that ablation performed in flowing liquid increases both productivity and reproducibility compared to stationary liquid. For this purpose, these authors have built an ablation cell in which the liquid flowed along the target surface. They explained their results by the fast removal of NPs and bubbles during the ablation procedure, which has been shown to be crucial at high repetition rate.

In this paper, we propose a cell design based on the horizontal irradiation of a cylindrical metal target and stirring configuration coaxially arranged with the target. After having assessed the repeatability of the properties of the colloids properties obtained under optimized conditions (standard procedure), we investigated the influence of the liquid stirring on the characteristics and repeatability of the generated Ag colloids. Additionally, the evolution of the width of the laser-machined groove (LMG, hereafter) was studied in stationary and stirred liquid and put in relation with the properties of the colloids.

\section{EXPERIMENT}

A schematic diagram of the experimental setup used in this work is shown in Figure 1. Laser ablation was carried out with the fundamental wavelength $(1064 \mathrm{~nm})$ of a NdYAG laser (Continuum Surelite I) delivering a maximum energy of $450 \mathrm{~mJ} /$ pulse with pulse duration of $6 \mathrm{~ns}$ (FWHM). Performing the experiment at the fundamental wavelength of the Nd-YAG laser also offers the best pulse-to-pulse stability conditions. This stability was further comforted by the use of an external energy attenuator (a $\lambda / 2$ plate in combination with a thin film polarizer), which enables the laser to run in optimized conditions of flash lamp energy and Q-switch delay. A particular attention has been devoted to the laser beam intensity distribution. The quality of the output beam can have a critical effect especially at low fluence in the vicinity of the ablation threshold. In our experiment, the spatial profile of the output beam was reshaped by means of an intracavity apodizer in order to attenuate the hot spots inherent to the elliptical pumping configuration of the laser rod. The beam intensity distribution in near-field has been checked with a profiling camera and is found to be rather uniform over $85 \%$ of the central part of the spot as shown in Figure 1(b). The diameter of the beam was $5 \mathrm{~mm}$. In order to maximize the ablation rate, the experiments were conducted at the maximum energy allowed by our system, i.e., $20 \mathrm{~mJ}$. Firstly, because of the proximity of the target to cell wall, excessive laser energy can cause damage on the cell wall. Furthermore, in the course of our experiments, we observed that a further increase of laser energy (typically above 25 $\mathrm{mJ})$ resulted in breaking of the cell. This may be due to the shock wave resulting from the plasma expansion in water which is accompanied by a significant release of energy. This energy limitation perhaps constitutes the main drawback of such an irradiation configuration.

A silver rod (Aldrich, $99.99 \%$ purity) of diameter $7 \mathrm{~mm}$ and length $10 \mathrm{~mm}$ was used as a target. Before each experiment, the silver target was polished with first a 4000 micromesh sand paper to remove the ablation grooves resulting from earlier experiments and then with a $0.1 \mu \mathrm{m}$ silica colloidal suspension to achieve a smooth surface. The target was finally cleaned in successive in ultrasonic baths with acetone, isopropanol, and deionized water (10 min each).

The metal target was mounted on a motorized stage, which allows simultaneous and independently controlled vertical translation and rotation around the target axis. The rotation speed of the target was set to $0.6 \mathrm{rpm}$ and its vertical translation to $0.6 \mathrm{~mm} \cdot \mathrm{min}^{-1}$, resulting in an ablation groove presenting a helical shape. 
The target was immersed in a $40 \mathrm{~mm}$ light-path borosilicate cell filled with $10 \mathrm{ml}$ ultra pure water $(18 \mathrm{M} \Omega \mathrm{cm})$. The colloidal solution was stirred during the ablation procedure by means of a stir rod of $9.5 \mathrm{~mm}$ diameter and $6 \mathrm{~mm}$ length (Cell spin bar, Fisherbrand) precisely placed just underneath the target and spun up by means a magnetic stirrer enabling rotation speeds from 0 to $1000 \mathrm{rpm}$. The laser beam was focalized on the target surface through the entrance face of the cell and the water by means of a plano-convexe borosilicate lens of $30 \mathrm{~mm}$ focal length. The distance covered by the laser beam in the water was $12 \mathrm{~mm}$. The spot diameter of the laser beam on the target was fixed to $500 \mu \mathrm{m}$ by precisely varying the position of the lens with respect to the surface of the target by means of a linear micrometer stage. Its diameter was measured in air by irradiating a silicon wafer positioned in place of the surface target at $20 \mathrm{~mJ}$. The duration of the ablation experiment was $300 \mathrm{~s}$ in all experiments.

Except for the stirring speed, these experimental parameters were all kept constant in this study. These parameters are the result of a preliminary optimization study, based on both literature and our own experience, aimed to obtain in a repeatable way and a reasonable processing time a colloidal solution showing a maximum extinction value close to 1 . In the following, we will refer to these optimized conditions as "standard procedure." Strong efforts have been made to maximize positions accuracy of the different mechanical and optical elements (quartz cell, target, and lens) constituting the PLAL system. This was performed by minimizing the degrees of freedom and/or even permanently fixing the position of several constitutive elements of the system such as the ablation cell, the magnetic stirrer, the transversal position of the focusing lens, the relative position of the axis of the target, and the stirrer. When displacements were required, such as the longitudinal position of the lens, they were ensured by micrometric linear stages.

The size distribution and morphology of the NPs were measured by means of a Transmission Electron Microscope (JEOL JEM-200 S) operated at $100 \mathrm{kV}$. Immediately after the experiment, a drop of the colloids was deposited on a coated copper grid and let dried in air. Each size distribution was obtained by measuring the maximum Feret diameter of about 400-500 particles using "ImageJ" freeware. ${ }^{19}$ The aspect ratio was obtained by measuring the ratio of the maximum to the minimum Feret diameter. The irradiated targets were examined by means of a scanning electron microscope (JEOL JSM-6390). The extinction spectra were recorded immediately after each experiment by means of a single beam UV-visible spectrophotometer (Libra S12, Biochrom) equipped with a quartz cell of $10 \mathrm{~mm}$ light path. The tolerance of the extinction, measured at $546 \mathrm{~nm}$ using neutral density standards is less than $1 \%$ and that of the wavelength $2 \mathrm{~nm}$.

\section{RESULTS}

\section{A. Influence of liquid stirring on the repeatability of the experiment}

The characteristics of the extinction spectra of colloids are considerably affected by the concentration, size, shape, and aggregation level of the NPs. Therefore, UV-visible spectroscopy is a useful tool to study, in a first approach, the influence of the experimental parameters on the NPs population. Figure 2 compares a set of four extinction spectra of colloidal solutions prepared according to the standard procedure with a stirring speed of $600 \mathrm{rpm}$ (Fig. 2(a)) and in stationary liquid (Fig. 2(b)). Visually, the colloidal solutions produced in stirred liquid presents a very similar and repeatable clear yellow-green colour and were stable for several weeks. This observation is confirmed by the extinction spectra which are found to be very similar suggesting a high degree of repeatability of the experiments in these experimental conditions.

The surface plasmon resonance (SPR) band is localized at $\lambda_{\max }=390 \mathrm{~nm}$ and has an average intensity of $1.05 \pm 0.10$. All the measurement uncertainties were evaluated according to the Student's t-distribution with a confidence interval of $95 \%$ for a set of 4 samples. The calculated standard deviation $\sigma_{\mathrm{n}-1}$ (where $\mathrm{n}-1$ represents the degrees of freedom) was further multiplied by 2.3534 to obtain the uncertainty value. The full-width at half maximum (FWHM) of the SPR band, which is related to the polydispersity in size and/or shape of the colloid, has a mean value of $55 \pm 7 \mathrm{~nm}$. The total amount of ablated material, evaluated from the extinction value at $250 \mathrm{~nm},{ }^{9}$ was found to be $0.30 \pm 0.04$ suggesting again that the ablation efficiency is repeatable from one experiment to
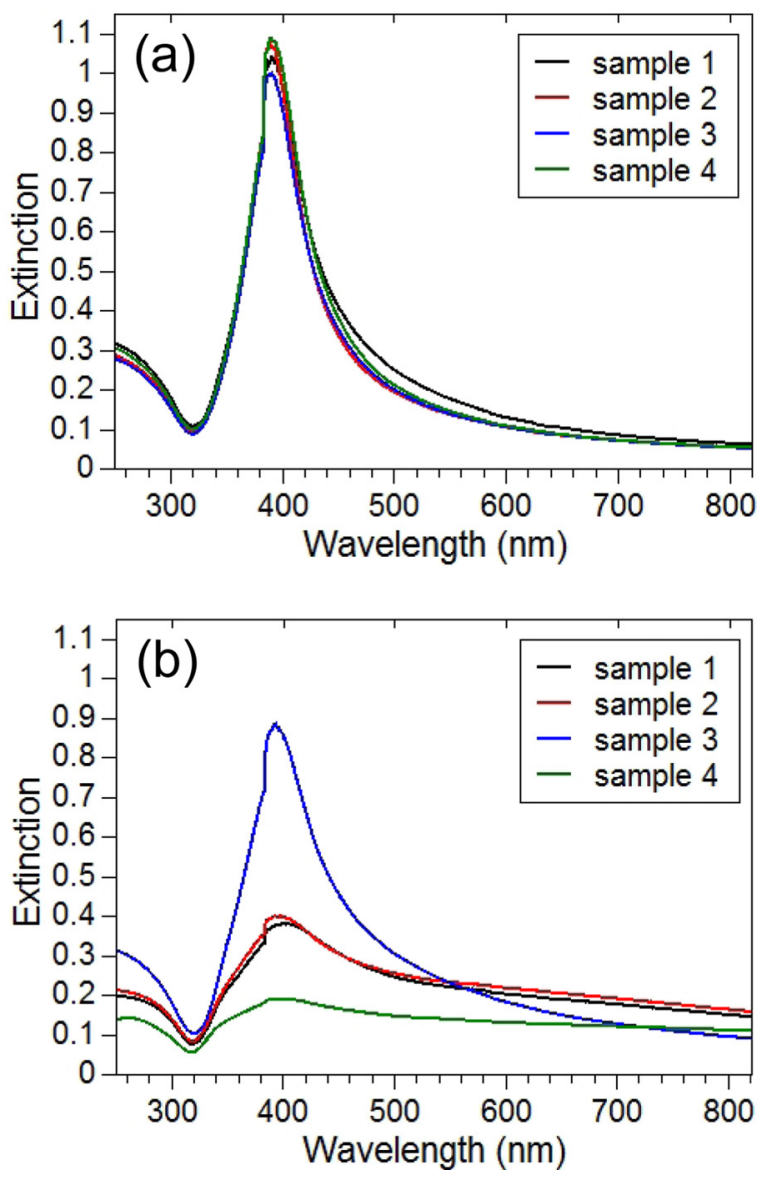

FIG. 2. UV-visible extinction spectra of four Ag colloids samples successively prepared according to the standard procedure at $600 \mathrm{rpm}$ (a) and in stationary liquid (b). 
the other. The colloids produced in stationary liquid were lighter than those obtained in stirred liquid with a more or less pronounced grey tint (depending on the considered sample). As shown in Fig. 2(b), their spectral characteristics were found to be unpredictable. In comparison with the experiments conducted in stirred liquid, the SPR band is wider by at least a factor 2 and its position is shifted to longer wavelength, i.e., $400 \mathrm{~nm}$. The amount of ablated silver and the SPR band intensity were on average considerably reduced by, respectively, 30\% and 60\%. During the course of the experiment, a visual inspection of the solution showed diffusion patterns, consisting of layers and filaments, typical of those observed as ink drops into water. By contrast, as the colloids solution was stirred, the colour of the colloids solution appeared immediately homogeneous and became denser as ablation proceeded.

These results show that the repeatability of the colloids properties is clearly improved when the liquid is stirred upon laser ablation. This is confirmed with the NPs size distributions obtained from TEM images of the NPs produced in stirred and stationary liquids and shown in Figure 3.
The NPs generated in stirred liquid are well-separated and mainly spherical even though a significant part of the population appears slightly elongated (Fig. 3(a)). Their size distribution, as visible in Fig. 3(b), is quite narrow and can be satisfactorily fitted by a log-normal function from which a mean diameter of $16 \pm 2 \mathrm{~nm}$ is found. The aspect ratio distribution (Fig. 3(c)) is also narrow and shows a mean value of $1.22 \pm 0.05$.

By contrast, the colloids generated under stationary liquid appear more heterogeneous in size and shape as shown by the TEM micrograph of Fig. 3(d). Spherical NPs are also observed but have larger diameter (up to $150 \mathrm{~nm}$ ) than those of Fig. 3(a). Furthermore, a significant part of the particles population consists of interconnected NPs forming chain-like structures with lengths reaching 100 to $200 \mathrm{~nm}$. As a consequence, the size distribution (Fig. 3(e)) appears broader and deviates from a log-normal function for Feret diameters exceeding $60 \mathrm{~nm}$. The mean particles size is found to be more than twice as large as those generated in stirred liquid, i.e., $28 \pm 5 \mathrm{~nm}$. Though, the aspect ratio distribution (Fig. 3(f)) is
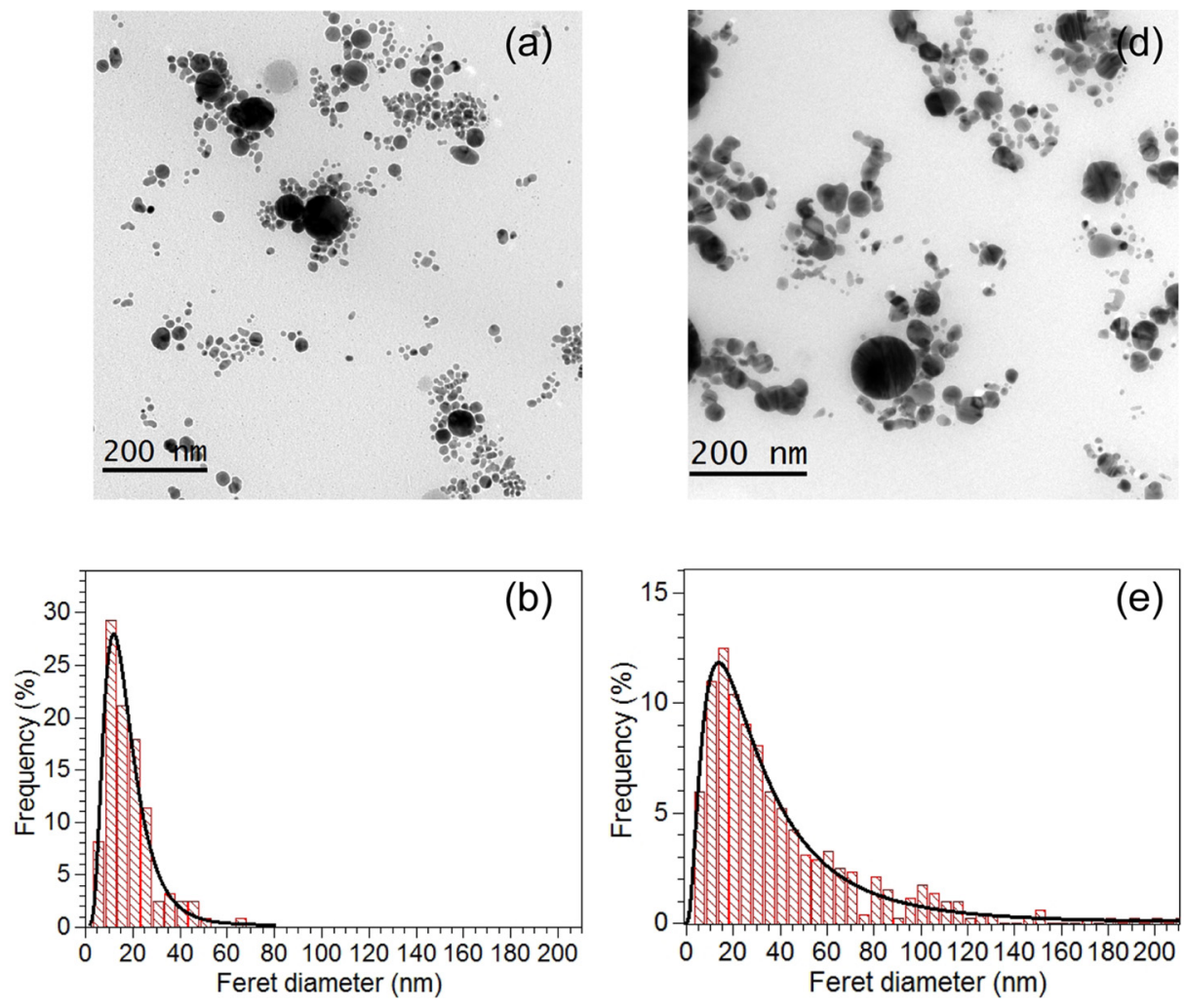

FIG. 3. Representative TEM image (a), size distribution (b) distribution of the aspect ratio (c) of the nanoparticles produced according to the standard procedure in stirred liquid at $600 \mathrm{rpm}$. Representative TEM image (d), size distribution (e) distribution of the aspect ratio (f) of the nanoparticles produced in stationary liquid. The solid lines represent the fit of the data by using a single log-normal function.
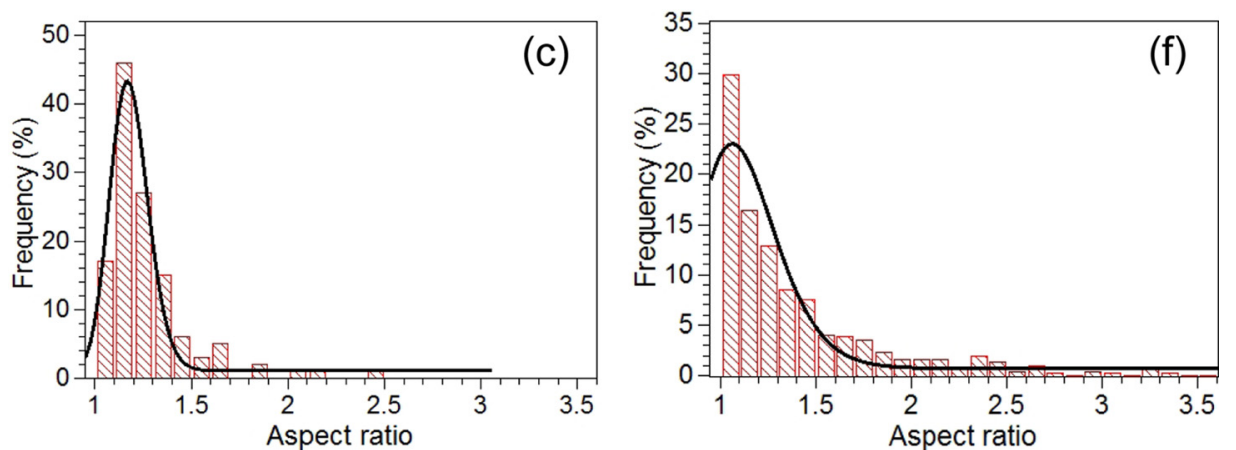
different, the mean value $1.25 \pm 0.22$ is found to be comparable to that of the colloids obtained in stirred liquid.

\section{B. SEM examinations of the laser-machined grooves on the targets}

To develop a better understanding of the laser interaction with the target during ablation and its relationship with the NPs population, the laser-machined grooves were systematically examined by SEM. Figure 4 presents a representative SEM micrographs showing the laser-machined groove on the Ag targets ablated in stirred (Fig. 4(a)) and stationary liquid (Fig. 4(b)). In stirred liquid, the grooves present sharp edges and their visual aspect is highly repeatable from one experiment to the other. Furthermore, no trace of material ejection at the micrometer scale was observed. High magnification SEM micrographs (not presented here) show that ridges and submicrometer scale droplets and particles were the dominant morphological features. In stationary liquid, the early stage of the groove is comparable to that of Fig. 4(a) but after few tens of seconds, it becomes irregular and increasingly narrow as the ablation proceeds.

The normalized evolutions of the grooves widths corresponding to samples 1, 2, 3, and 4 of Figs. 2(a) and 2(b) have been plotted against laser exposure time in Figs. 4(c) and 4(d), respectively. In stirred liquid (Fig. 4(c)), the grooves widths are found to decay smoothly and almost linearly with almost the same decay rates. At the end of the experiment ( $300 \mathrm{~s})$, the width of the groove was reduced by $23 \%$ on average compared with its initial value. In stationary liquid Fig. 4(d), the tracks left by the laser pulses are not repeatable from one experiment to the other and presents in most cases an exponential decay. It appears, moreover, that there is a correlation between the decay rate of the groove width and the extinction spectra (Fig. 2(b)). The larger the decay rate of the groove width, the smaller are the colloids concentration and the intensity of the plasmon band and the larger is the FWHM. It is noted that, after $300 \mathrm{~s}$ laser exposure time, the groove width decreases by $30-35 \%$ of its initial value. These findings strongly suggest that the laser energy reaching the target surface diminishes with exposure time due to absorption/scattering of the incident laser beam by the colloids. These results also reveal a correlation between the repeatability of the time evolution of the groove width and the repeatability of the colloids properties.

\section{DISCUSSION}

\section{A. Quantification of the laser light absorption by the colloid during laser ablation}

The gradual attenuation of the laser-machined groove can be explained by the increasing absorption and/or scattering of the laser beam during the processing time, leaving less energy reaching the target surface. In order to quantify the laser energy attenuation, an Ag target was irradiated in water according to our standard procedure but only for few seconds and at various energies values from 1 to $20 \mathrm{~mJ}$ and the resulting groove width was measured. The water was renewed between each experiment. Figure 5 presents the evolution of the initial width of the laser-machined groove as a function of laser energy.

Three relevant regions of interest noted I, II, and III and delimited by dashed lines in Fig. 5 are distinguishable. Region I corresponds to energy below the ablation threshold where no visible trace of matter removal was observed on the target surface. Region II, between 2 and $5 \mathrm{~mJ}$, corresponds to an energy interval surrounding the ablation threshold which is estimated to be around 2-3 mJ. In this latter case, a SEM examination of the surface showed scattered ablation traces mainly localized on the polishing marks and scratches of the virgin surface. In this case, the groove edge was not sufficiently marked to permit any width measurement. For region III, a uniform and
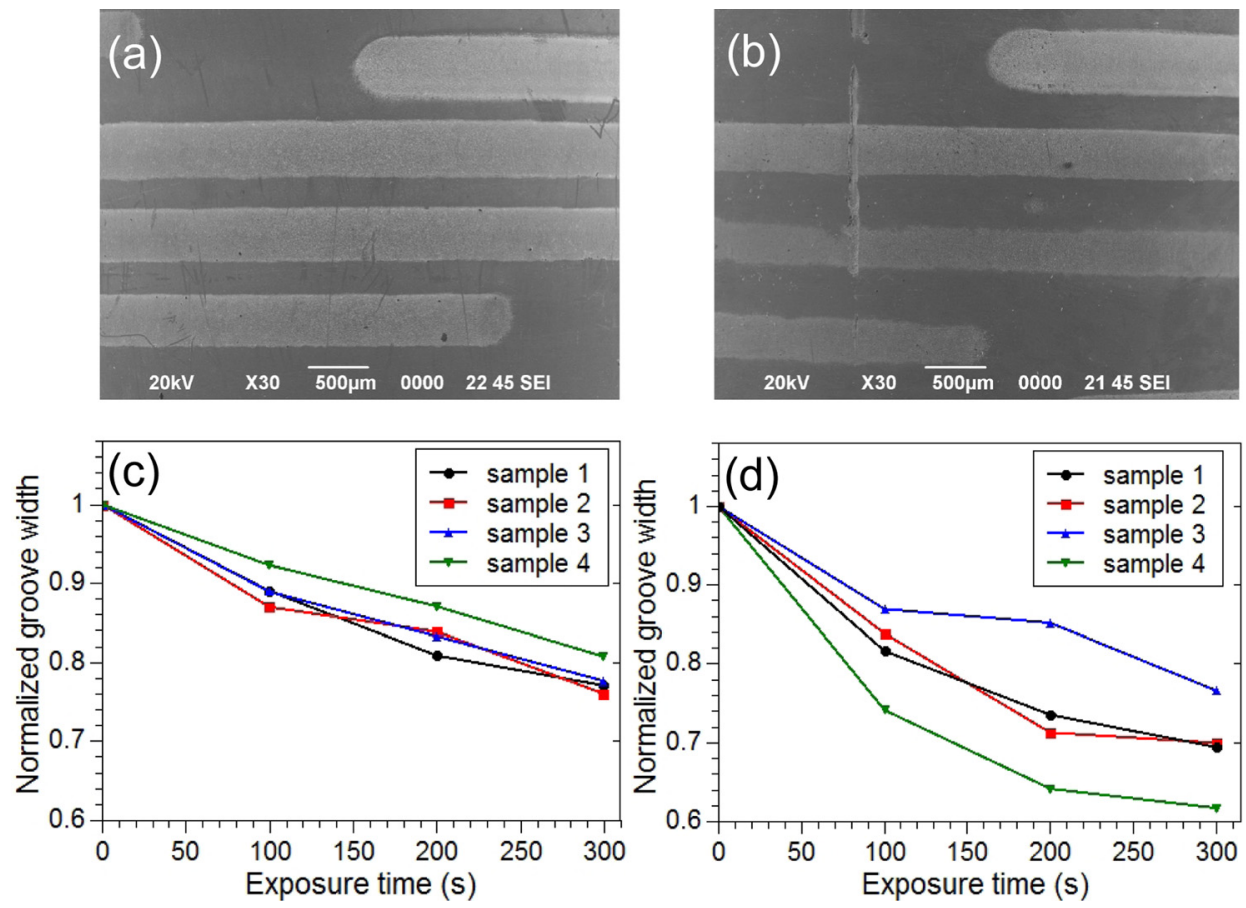

FIG. 4. SEM images of a representative laser-machined groove (LMG) on an $\mathrm{Ag}$ target ablated in stirred liquid at $600 \mathrm{rpm}$ (a) and in stationary liquid (b). Evolution of the width of the lasermachined groove as a function of laser exposure time in stirred liquid at $600 \mathrm{rpm}$ (c) and in stationary liquid (d). The grooves widths were measured from the SEM images of the irradiated Ag targets corresponding to the samples presented in Fig. 2. 


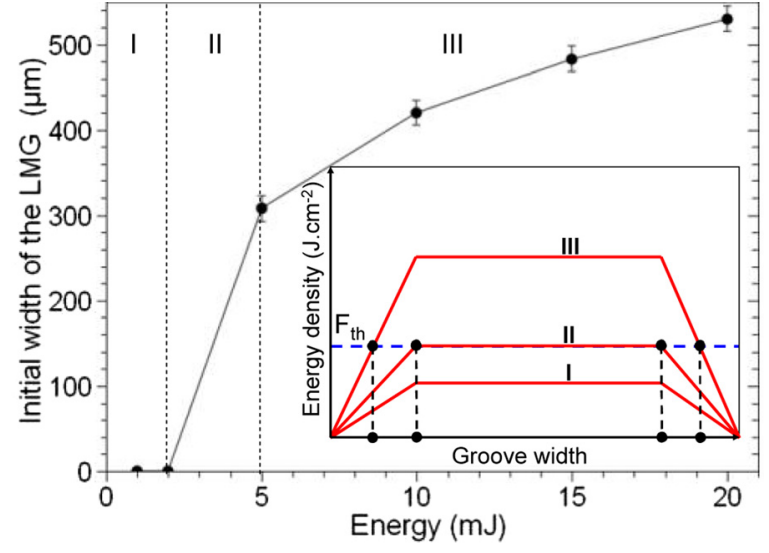

FIG. 5. Initial width of the grooves obtained from SEM micrographs of a silver target irradiated at various laser energies. The irradiations were performed in water during an exposure time of $20 \mathrm{~s}$. The inset represents the relationship between the width of the groove and the intensity distribution of the laser (red lines) for the 3 regions indicated in the graph. The horizontal blue line represents the ablation threshold $\left(\mathrm{F}_{\mathrm{th}}\right)$ of the silver target.

clearly delimited groove (as in Fig. 4(a)), whose initial width gently evolves from $300 \mu \mathrm{m}$ up to $520 \mu \mathrm{m}$ as the energy increases from 5 to $20 \mathrm{~mJ}$ can be appreciated. The evolution of the surface topography and that of the initial width of the groove with laser energy are consistent with the beam intensity profile shown in Fig. 1(b). The inset in Fig. 5 schematically indicates the relationship between the intensity profile and the groove width. The latter corresponds to the intersection of the intensity distribution curves with the ablation threshold of the target $\left(\mathrm{F}_{\mathrm{th}}\right)$ which is represented by a horizontal dashed blue line. For region I, the maximum of the intensity distribution is clearly below $\mathrm{F}_{\text {th }}$ and there is no material removal. Close to $F_{\text {th }}$, the ablated area results from the fluctuations of the intensity distribution plateau that surpass the ablation threshold and/or the surface defects. For region III, abscises of the intersection points between the intensity distribution curve and $F_{t h}$ determine the groove width. Using Fig. 5 , it is possible to determine to the part of incident laser energy that effectively reaches the target surface and thus the attenuation of the incident laser energy by the colloids.

In stirred liquid, the initial groove width that is $520 \mu \mathrm{m}$ decreases by $23 \%$ (Fig. 4(c)) after $300 \mathrm{~s}$ ablation time reaching a value of $400 \mu \mathrm{m}$. One can deduce from Fig. 5 that the laser energy actually reaching the target is then of about 10 $\mathrm{mJ}$, i.e., an attenuation of $50 \%$ of the laser energy. In stationary liquid, after $300 \mathrm{~s}$, the groove width decreases by 30-35\% (Fig. 4(d)). In that case, a similar reasoning shows that the energy actually reaching the target drops to 6-7 $\mathrm{mJ}$, i.e., an attenuation of $65-70 \%$ of the laser energy.

\section{B. Possible origin of the laser light attenuation during the ablation process}

Due to stirring, we could assume, in a first approach that the generated NPs disperse in the bulk liquid and the extinction of the colloid solution increases continuously with ablation time explaining the continuous decrease of the energy reaching the target. However, the extinction value of the bulk colloidal solution at $1064 \mathrm{~nm}$ is not high enough to explain such strong attenuations. In stirred liquid after $300 \mathrm{~s}$, the extinction coefficient at $1064 \mathrm{~nm}$ of the produced colloids is $\sim 10^{-2}$ (Fig. 2(a)) which would give rise to an attenuation of only $2-3 \%$ for a light path of $10 \mathrm{~mm}$. Let us recall here that the distance covered by the laser beam during the laser processing is comparable, i.e., $12 \mathrm{~mm}$.

The absorption of the laser beam by cavitation gas bubbles $^{18}$ can also not be invoked. In experimental conditions close to those described in this work, the cavitation gas bubble lasts few hundreds $\mu \mathrm{s} .{ }^{20}$ At a repetition rate of $10 \mathrm{~Hz}$, the time which elapses between two consecutives pulses, i.e., $0.1 \mathrm{~s}$, is two or three order magnitude longer than the lifetime of the cavitation bubble.

Tsuji et al. ${ }^{10}$ distinguish two possible mechanisms for the absorption of the laser beam upon the ablation process. The first one designed by the authors as "intra-pulse" mechanism corresponds to the attenuation of the later part of a pulse by the NPs formed upon the leading part of the same pulse. The second one "inter-pulse" mechanism for which the NPs formed upon a given pulse attenuates the consecutive pulses. The former mechanism is consequently independent of the ablation time and thus cannot explain the decay of the width of the laser-machined groove which would have otherwise been constant. By contrast, the second mechanism appears to be more relevant to justify this evolution. Since the characteristic time for NPs diffusion is much larger than the time that elapses between two consecutive pulses $\left(10^{-1} \mathrm{~s}\right)$, one can reasonably assume that the incident laser beam was attenuated by an accumulation of NPs forming a more or less optically thick layer in front of the target $^{10,11,21}$ during the ablation process. This dense NPs layer gains NPs upon multi-pulses ablation $(10 \mathrm{~Hz})$ of the target and undergoes losses through (i) NPs diffusion in the bulk solution due to density gradients, (ii) convection due to thermal gradient, ${ }^{22}$ and (iii) forced (mechanical) convection due to stirring.

Therefore, we suggest that the decrease of the width of the groove (Fig. 4) reflects a gradual increase of the absorption of the laser beam by dense NPs layers in the vicinity of the target surface which optical thickness increases monotonously with exposure time.

\section{Relationships between the colloids characteristics, the laser light attenuation, and the stirring conditions}

To develop a better understanding of the connection between the evolution of the groove and liquid stirring, we propose a scenario based on mass transfer sketched in Figure 6.

In stirred liquid (Fig. 6(a)), the circulatory motion induced by the stir rod is expected to quickly disperse the NPs from the surface target in the $\mathrm{x}$ direction due to forced convection (diffusion is reasonably assumed to be negligible in this direction). As a consequence of friction or viscous effects, a boundary layer is expected to be formed on the target surface and the mass transfer in the y direction (normal direction of the target) is essentially diffusive. This boundary layer is then restricted to a confined area, close to the target surface, where the laser fluence is expected to be high enough to provoke NPs fragmentation. ${ }^{9,14,23}$ 
(a) Stirred liquid

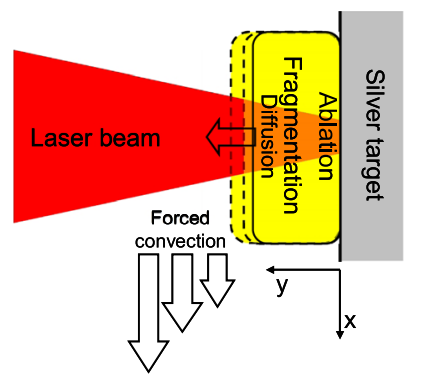

(b) Stationary liquid

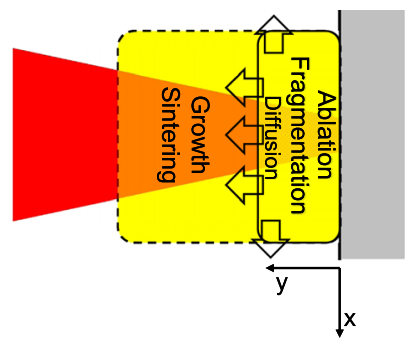

FIG. 6. Schematic diagram representing the mass transfer of the colloids in the vicinity of the target surface in (a) stirred liquid and (b) stationary liquid and the consequences of the interaction of the NPs with the laser beam.

In stationary solution (Fig. 6(b)), the produced NPs expand mainly in the $y$ direction at greater distance from the target surface as a consequence of diffusion (Brownian motion) and the thermal convection flows. ${ }^{22}$ Part of the NPs formed and fragmented near in the vicinity of the target are transferred in the y direction and may reach a part of the laser beam where the laser fluence becomes not sufficient to provoke the entire melting and fragmentation of the NPs. Boyer and Meunier ${ }^{24}$ have studied the influence of solvent on the NPs growth from light absorption by colloids. They have performed nanosecond laser annealing at $532 \mathrm{~nm}$ for $30 \mathrm{~min}$ at $10 \mathrm{~Hz}$ in methanol with an unfocused beam on $\mathrm{Au}$ NPs that were previously synthesized by laser fragmentation. They observed an increase of the NPs size they attributed to a diffusion coalescence process. The same mechanism can be invoked to explain the results obtained in stationary liquid. The heated and/or partially melted particles gain kinetic energy causing increase of their collision frequency and the growth and/or sintering ${ }^{21}$ of NPs explaining the formation of large size NPs and/or chain-like structures as shown in Fig. 3(d). These latter contribute significantly for the red shift of the plasmon band and the red tail observed in Fig. 2(b) that is related to a convolution of the bands associated with the longitudinal modes of their surface plasmon ${ }^{25}$ in addition to light scattering by large spherical NPs. These particles would be then responsible for the enhanced extinction of the laser beam during ablation. Furthermore, the stochastic nature of this mass transfer process and thus that of the amount of NPs interacting with the beam also explain the poor repeatability of the properties of the colloids obtained in stationary liquid.

\section{CONCLUSION}

An ablation cell for PLAL experiments based on the horizontal irradiation of a cylindrical metal target with wellcontrolled stirring configuration was described and critically assessed. Pulsed-laser ablation in so stirred liquid shows advantages over stationary liquid by greatly increasing the ablation yield (by 30\%) and considerably improving the repeatability and the quality of the colloids properties.
Additionally, this system enabled to establish a clear relationship between the laser-machined groove and the quality of the generated colloids. A careful examination of the target after ablation process revealed a decay of the width of the laser-machined groove with ablation time. We showed that this decay is due to a local accumulation of NPs in front of the target that attenuates the incident laser energy and is responsible for the decrease of ablation yield with exposure time. These results strongly suggest that the observed influence of stirring on the NPs population and its repeatability is related to the mass transfer of the generated NPs in the bulk solution and the interaction of the NPs with the laser beam. We believe that the control of mass transfer is crucial in PLAL experiments and deserves further theoretical and experimental studies.

${ }^{1}$ E. Boisselier and D. Astruc, Chem. Soc. Rev 38, 1759 (2009).

${ }^{2}$ E. C. Dreaden, A. M. Alkilany, X. Haung, C. J. Murphy, and M. A. ElSayed, Chem. Soc. Rev 41, 2740 (2012).

${ }^{3}$ P. Liu, C. Liang, X. Ling, C. X. Wang, and G. Yang, ACS Nano 5, 4748 (2011).

${ }^{4}$ J. Neddersen, G. Chumanov, and T. M. Cotton, Appl. Spectrosc. 47, 1959 (1993).

${ }^{5}$ P. Liu, H. Cui, C. X. Wang, and G. W. Yang, Phys. Chem. Chem. Phys. 12, 3942 (2010).

${ }^{6}$ S. Barcikowski and G. Compagnini, Phys. Chem. Chem. Phys. 15, 3022 (2013).

${ }^{7}$ V. Amendola and M. Meneghetti, Phys. Chem. Chem. Phys. 11, 3805 (2009).

${ }^{8}$ J. P. Sylvestre, S. Poulin, A. V. Kabashin, E. Sacher, M. Meunier, and J. H. T. Luong, J. Phys. Chem. B 108, 16864 (2004).

${ }^{9}$ F. Mafuné, J. Kohno, Y. Takeda, T. Kondow, and H. Sawabe, J. Phys. Chem. B 104, 9111 (2000).

${ }^{10}$ T. Tsuji, K. Iryo, N. Watanabe, and M. Tsuji, J. Photochem. Photobiol. A 145, 201 (2001).

${ }^{11}$ T. Tsuji, K. Iryo, N. Nishimura, and M. Tsuji, Appl. Surf. Sci. 202, 80 (2002).

${ }^{12}$ M. A. Sobhan, M. Ams, M. J. Withford, and E. M. Goldys, J. Nanopart. Res. 12, 2831 (2010).

${ }^{13}$ C. L. Sajti, R. Sattari, B. Chichkov, and S. Barcikowski, J. Phys. Chem. C 114, 2421 (2010).

${ }^{14}$ M. Prochàzka, P. Mojzes, J. Stepanek, B. Vlckova, and P.-Y. Turpin, Anal. Chem. 69, 5103 (1997).

${ }^{15}$ A. Pyatenko, K. Shimokawa, M. Yamaguchi, O. Nishimura, and M. Suzuki, Appl. Phys. A 79, 803 (2004).

${ }^{16}$ A. Menéndez-Manjón, B. Chichkov, and S. Barcikowski, J. Phys. Chem. C 114, 2499 (2010).

${ }^{17}$ Y. H. Chen and C. S. Yeh, Colloids Surf. A 197, 133 (2002).

${ }^{18}$ S. Barcikowski, A. Menéndez-Manjón, B. Chichkov, M. Brikas, and G. Račiukaitis, Appl. Phys. Lett. 91, 083113 (2007).

${ }^{19}$ See http://rsbweb.nih.gov/ij/index.html for "ImageJ, Image Processing and Analysis in Java," Wayne Rasband. National Institutes of Health, USA.

${ }^{20}$ P. Wagener, S. Ibrahimkutty, A. Menzel, A. Plech, and S. Barcikowski, Phys. Chem. Chem. Phys. 15, 3068 (2013).

${ }^{21}$ Z. Yan, R. Bao, Y. Huang, A. N. Caruso, S. B. Qadri, C. Z. Dinu, and D. B. Chrisey, J. Phys. Chem. C 114, 3869 (2010).

${ }^{22}$ G. Compagnini, E. Messina, R. S. Catliotti, A. Grillo, and G. Giaquinta, Philos. Mag. Lett. 89, 250 (2009).

${ }^{23}$ F. Mafuné, J. Kohno, Y. Takeda, and T. Kondow, J. Phys. Chem. B 106, 7575 (2002).

${ }^{24}$ P. Boyer and M. Meunier, J. Phys. Chem. C 116, 8014 (2012).

${ }^{25}$ S. Link and M. A. El-Sayed, J. Phys. Chem. B 103, 8410 (1999). 